\title{
Neuroprotective Benefits of $S$. platensis Extract on Drosophila melanogaster Model of Parkinson's Disease
}

\author{
Mohamad Agus Salim ${ }^{1 *}$, Muhammad Subandi ${ }^{2}$, Yeni Yuniarti ${ }^{3}$ \\ \{ agus.salim@uinsgd.ac.id ${ }^{1}$, mhd.subandi@uinsgd.ac.id ${ }^{2}, Y e n i \_Y u n i a r t i @ u_{\text {upi.edu }}^{3}$ \} \\ ${ }^{1}$ Biology Department of Science and Technology Faculty of \\ UIN Sunan Gunung Djati Bandung, 40614, Bandung, Indonesia \\ ${ }^{2}$ Agrotechnology Department of Science and Technology Faculty of \\ UIN Sunan Gunung Djati Bandung, 40614, Bandung, Indonesia \\ ${ }^{3}$ Mathematic Education of Universitas Pendidikan Indonesia,40154, \\ Bandung, Indonesia.
}

\begin{abstract}
Blue-green microalgae S. platensis has been known for a long time as a source of food with high nutritional value and pharmacological properties. In addition to high protein content, it also contains high antioxidant compounds including total polyphenols, which indicate the possibility of functioning as a pharmaco-therapeutic utility. In the current study, we have demonstrated the neuroprotective effect of $S$. platensis extract on the survival rate and locomotion capability. Using wild type of male fruit flies (Drosophila melanogaster) were exposed to $\mathrm{FeSO}_{4}(15 \mathrm{mM})$ and/or $S$. platensis extract $(120 \mu \mathrm{g} / \mathrm{mL})$ supplemented in their diet for 4 days of study. The results showed that dietary supplementation of $S$. platensis extract significantly increase the survival rate, locomotor activity, dopamine compound and decrease in malondialdehyde levels of $\mathrm{FeSO}_{4}$-fed fruit flies. This indicates the involvement of polyphenol as antioxidant compounds contained in $S$. platensis extract. From our results it is concluded that $S$. platensis extract was effective in decreasing the toxicity of $\mathrm{FeSO}_{4}$ in fruit flies as well as confirm the utility of this model to observe potential therapeutic on neurodegenerative disorders, especially Parkinson's disease.
\end{abstract}

Keywords: $\mathrm{FeSO}_{4}$, Fruit fly, Parkinson, Spirulina Extract.

\section{Introduction}

Parkinson's disease is characterized by the degeneration of dopaminergic neurons in the substantia nigra, which is in the basal ganglia region where it produces and stores dopamine neurotransmitters [1]. The clinical symptoms of Parkinson's disease are movement abnormalities caused by decreased dopamine compounds and increased iron levels in the striatum [2]. Incorrect iron micronutrient metabolism as one of the emergence of oxidative stress causes of neurodegenerative disorder such as Parkinson's disease that create abnormal neurobehavioral functions [3]. According to [4] iron has an important role in maintaining normal physiological functions of the brain such as mitochondrial respiration and synthesis of dopamine compound. Iron has a function as a catalyst in reactions that involve transferring electrons together with oxygen and hydrogen peroxide producing hydroxyl radicals that are very reactive 
and destructive [5]. Iron metabolism produces a lot of reactive oxygen species (ROS) causing lipid peroxidation observed in Parkinson's disease [6].

At present the treatment of Parkinson's disease aims to reduce the appearance of symptoms and focus on the lack of dopamine levels [7]. One alternative in the treatment of Parkinson's disease is the use of S. platensis [8]. For a long time, S. platensis was used as a food supplement because it contains complete nutrients needed by the human body. S. platensis is a blue green microalgae (cyanobacteria) that can produce polyphenolic compounds as predominant antioxidant [9]. The research conducted by [10] explained that polyphenol compounds were able to maintain locomotor organ function in iron-induced fruit fly.

Fruit flies are insects that can live anywhere, easily breed with a short life cycle so they are cheaper to maintain. According to [11] the use of fruit flies offers the advantage of faster drug screening analysis that is not obtained when using animal models from the mammal group. In this paper we try to explain how to find out the levels of polyphenol compounds and the strength of antioxidant activity contained in S. platensis extract through free radical scavenging assay. The physiological process in the fruit fly brain that has been treated with FeSO4 and $S$. platensis extract is also recorded from the content of malondialdehide and dopamine. Likewise, the importance of the treatment effect on survival and locomotor phenotypes of fruit flies as a characteristic of Parkinson's disease will be described.

\section{Materials and Methods}

\subsection{Culture and harvest of microalgae $S$. platensis}

The microalgae used in this study were the $S$ platensis species purchased from School of Life Science and Technology ITB, Bandung Indonesia. Furthermore, S. platensis was cultured on F/2 medium to obtain the expected amount of biomass. Propagation culture on plastic containers with a capacity of $10 \mathrm{~L}$. Culture room with day / night temperature $27 / 21{ }^{\circ} \mathrm{C}$ and lighting using TL 45 watts lamp with an intensity of 2500 Lux throughout the day until the end of the exponential growth phase on day 10. Microalgae are harvested using filtration on cloth monyl 300 messes. Microalgae biomass was dried at room temperature under a 25 watt TL lamp for 24 hours. Finally dry biomass was crushed using mortal and ready to be extracted.

\subsection{Preparation of $S$. platensis extract}

Dry biomass from S. platensis was prepared $4 \mathrm{~g}$ to be macerated with $20 \mathrm{~mL}$ of methanol solution p.a. Then the mixture was placed in the shaker incubator for 24 hours to remove the active compound from S. platensis biomass. Then the solution was filtered using filter paper to separate the filtrate and residue. Methanol solvents from the filtrate were removed by evaporating in the water bath so that the crude extract in the form of concentrated paste was obtained.

\subsection{Determination of total polyphenol content}

The total polyphenol compound content was determined using the calorimetric method according to [12]. One g of S. platensis biomass powder is dissolved in $10 \mathrm{ml}$ of $80 \%$ methanol. 
The extract $(1 \mathrm{ml})$ was shaken for $30 \mathrm{~min}$ and centrifuged at $2000 \mathrm{x}$ g for $15 \mathrm{~min}$. The extract was taken to be dissolved with $1 \mathrm{ml}$ of Folin-Ciocalteau reagent (Folin reagent dissolved with distilled water 1: 10) and with $2 \mathrm{ml}$ of $7.5 \% \mathrm{Na}_{2} \mathrm{CO}_{3}$ solution. After the solution was left for 20 minutes, the absorbance is measured at $765 \mathrm{~nm}$ using a Spectrophotometer UV-Vis Cary 60 is against aquadest as blank. As a standard, gallic acid was used and total polyphenol were evaluated using a calibration curve.

\section{4 $\mathrm{DPPH}$ radical-scavenging activity}

Measurement of antioxidant activity using the DPPH method (2,2-diphenyl-1picrylhydrazyl) free radical reduction and vitamin C as a control [13]. S. platensis extract was dissolved in methanol solution with final concentrations of 40, 80, 120, 160 and $200 \mu \mathrm{g} / \mathrm{mL}$. Then added $1.0 \mathrm{~mL}$ of DPPH and methanol solution to $5 \mathrm{~mL}$, homogenized and covered with aluminum foil. The sample and control solutions were incubated for $30 \mathrm{~min}$. at $37^{\circ} \mathrm{C}$. The absorbance value was measured at a wavelength of $516 \mathrm{~nm}$ using a UV-Vis spectrophotometer. Antioxidant activity expressed in \% inhibition determined by equation:

$$
\text { (Inbibition }(\%))=\frac{\text { Absorbance control }- \text { Absorbance extract }}{\text { Absorbance control }} \times 100 \%
$$

\subsection{Drosophila melanogaster culture}

The male fruit fly obtained was the result of cultivation that has been carried out previously and selected based on the same age. The medium of growing male fruit flies consists of a mixture containing $1 \% \mathrm{w} / \mathrm{v}$ yeast, $1 \% \mathrm{w} / \mathrm{v}$ sucrose, $1 \% \mathrm{w} / \mathrm{v}$ milk powder, $2 \% \mathrm{w} / \mathrm{v}$ agar, $0.8 \% \mathrm{w} / \mathrm{v}$ nipagin stored in a bottle culture. Fruit flies received treatment for 4 days and maintained at room temperature, lighting cycles with 12 hours of light and 12 hours of darkness.

\subsection{Survival rate}

In evaluating survival rates, fruit flies was counted in survivors, expressed in the percentage of fruit flies that still survive. This was intended to determine the effectiveness of each treatment given to fruit flies. Each treatment group used 90 fruit flies were reared in 3 culture bottles, so that there were 30 fruit flies per bottle. Calculations were carried out every day until the end of this observation (4 days).

\subsection{Negative Geotaxis Assay}

Negative geotaxis assay was a test of fruit fly locomotor ability based on the method of [14]. Fruit flies were anesthetized using cold water (ice) as soon as possible placed on the base of a vertical glass tube. The locomotoric phenotype of 10 fruit flies for each treatment was observed from the climbing ability with a height of $5 \mathrm{~cm}$ in $6 \mathrm{sec}$. (Figure.ure 1). The data obtained was presented in the form of a percentage. 


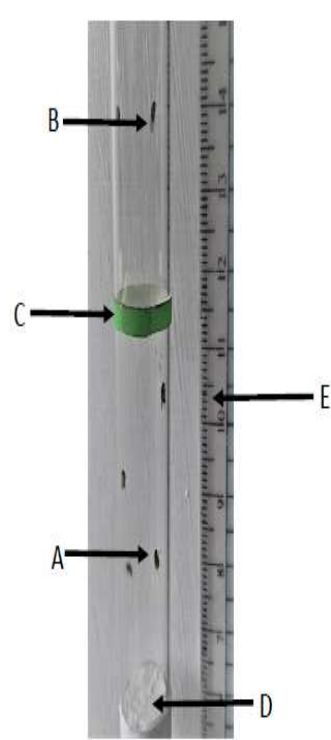

Fig. 1. Negative geotaxis assay of fruit flies (a) unable to pass (b) able to pass (c) boundary mark, $5 \mathrm{~cm}$ high (D) base of coloum tube (E) ruler scale

\subsection{Lipid Peroxidation Assay}

Measurement of lipid peroxidation based on the method of [15]. Then 30 heads of fruit flies were prepared to be homogenized in $0.6 \mathrm{~mL}$ of $50 \mathrm{mM}$ sodium phosphate buffer and $10 \%$ trichloroacetic acid (TCA) at $\mathrm{pH}$. Then centrifuged at $10,000 \mathrm{rpm}$ for $10 \mathrm{~min}$. The supernatant was divided into two aliquots: the first supernatant $(0.3 \mathrm{~mL})$ was mixed with $0.1 \mathrm{~mL}$ EDTA solution $0.1 \mathrm{M}$, and $0.6 \mathrm{~mL} 1 \%$ thiobarbiturate acid solution in $0.05 \mathrm{M} \mathrm{NaOH}$ solution. Then the mixture was incubated at $100^{\circ} \mathrm{C}$ for $15 \mathrm{~min}$. The second aliquot as a control $(0.3 \mathrm{~mL})$ was mixed with $0.7 \mathrm{~mL} \mathrm{H}_{2} \mathrm{O}$ and incubated at the same temperature and time as for the first aliquat. After being cooled on ice, the sample was centrifuged at 10,000 rpm for $1 \mathrm{~min}$. The malondialdehyde (MDA) product was measured at $\lambda 535 \mathrm{~nm}$.

\subsection{Dopamine Content Assay}

In this measurement, 40 heads of fruit flies were homogenized in $500 \mu \mathrm{L} \mathrm{HCl-butanol}$ $(0.85 \mathrm{~mL} 37 \% \mathrm{HCL}$ in $1 \mathrm{~L} \mathrm{n}$-butanol). The suspension was then centrifuged at $3000 \mathrm{rpm}$ for 5 min. The resulting supernatant was added $250 \mu \mathrm{L}$ heptane and $100 \mu \mathrm{L} 0.1 \mathrm{M} \mathrm{HCl}$ and centrifuged at $3000 \mathrm{rpm}$ for $5 \mathrm{~min}$. and the resulting supernatant was used for testing. The mixture of the test solution consisted of $100 \mu \mathrm{L}$ of supernatant, $50 \mu \mathrm{L}$ of $\mathrm{HCl} 0.4 \mathrm{M}$ and $100 \mu \mathrm{L}$ of iodine solution, left for $2 \mathrm{~min}$. at room temperature. After settling, $100 \mu \mathrm{L}$ sodium sulfite and $100 \mu \mathrm{L} 10 \mathrm{M}$ acetic acid were added, then the mixture was boiled at $100^{\circ} \mathrm{C}$ for $6 \mathrm{~min}$. The mixture was cooled at room temperature and measured at a wavelength of $375 \mathrm{~nm}$ [16].

\subsection{Statistical Analysis}


Statistical analysis was carried out using SPSS version 18. software program. The value of each data obtained was expressed as mean \pm standart deviation (SD). The significance for each data from all treatments was obtained from testing using one-way ANOVA. If there were significant $(\mathrm{p}<0.05)$ differences then analysis was followed by Duncan multiple range test.

\section{Results}

\subsection{Total polyphenols concentration and antioxidant activity}

Extract of $S$. platensis has been determined for antioxidant activity and polyphenol content. Total polyphenol concentration was measured by tiobarbiturate acid assay of $421.32 \mathrm{mg} \mathrm{GAE} / \mathrm{g}$. The antioxidant activity as measured by the DPPH test showed that $S$. platensis extract had the potential to scavenge DPPH free radicals. The IC50 value for extract of S. platensiswas 64.55 $\mu \mathrm{g} / \mathrm{mL}$ while ascorbic acid was $2.27 \mu \mathrm{g} / \mathrm{mL}$.

\subsection{Survival Rate}

The survival ability of fruit flies counted every 24 hours for four days of observation. From Figure.ure 2, the fruit fly group in the control (C) was not significantly different from the treatment of extract of $S$. platensis (SP). Whereas the FeSO4-induced fruit fly group showed the lowest survival. The double treatment of the fruit fly group which received extract of $S$. platensis and FeSO4 $(\mathrm{FE}+\mathrm{SP})$ proved the ability of extract $S$. platensis to counteract FeSO4 toxicity. It was evident that the fruit fly group that received only FeSO4 treatment (FE) showed the lowest survival.

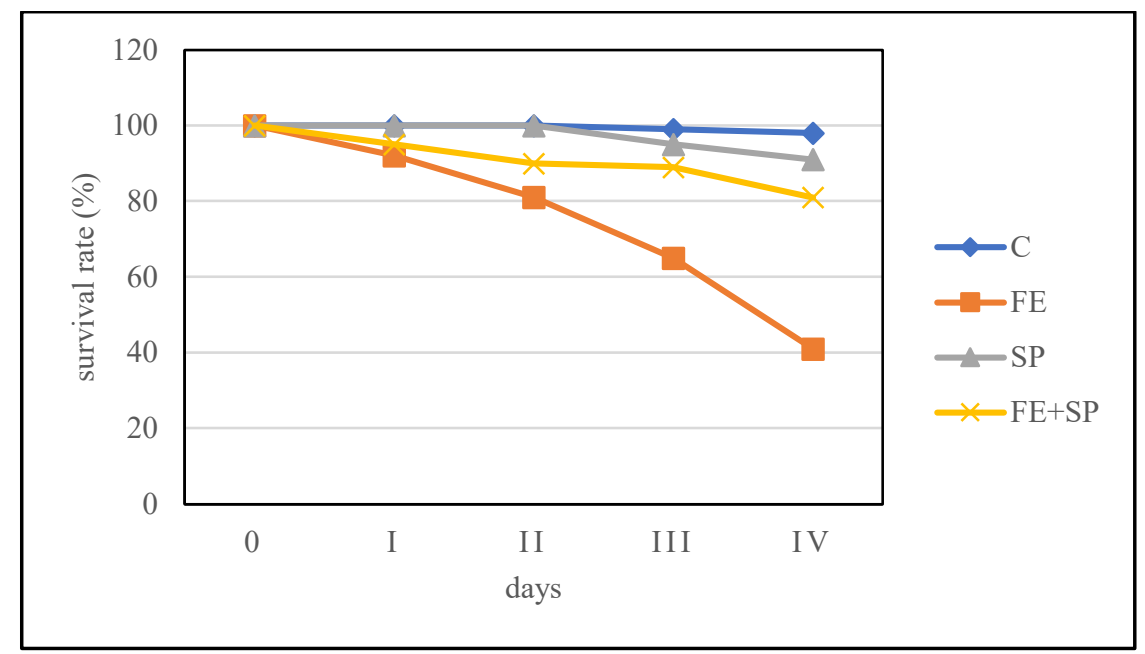

Fig. 2. Effect of SP $(120 \mu \mathrm{g} / \mathrm{mL})$ and FE $(3.5 \mathrm{mM})$ on survival ability of fruit flies during four days of observation.; Abbreviation of C: control, FE: FeSO4, SP: S. platensis extract. Data in the form of an average \pm Standard error. Values with different letters show significant differences at $\mathrm{p}<0.05$ (DMRT). 


\subsection{Negative Geotaxis}

FeSO4 treatment of fruit flies greatly influences climbing ability. It is seen that the fruit fly received FeSO4 treatment (FE-treatment) had the lowest value of the negative geotaxis assay. The treatment of $S$. platensis extract (SP-treatment) was able to reduce FeSO4 toxicity as indicated by higher locomotoric ability values in the double treatment between $\mathrm{FeSO} 4$ and extract of S. platensis (FE+SP-treatment) from a single treatment FeSO4 alone (FE-treatment) (Figure.ure 3).

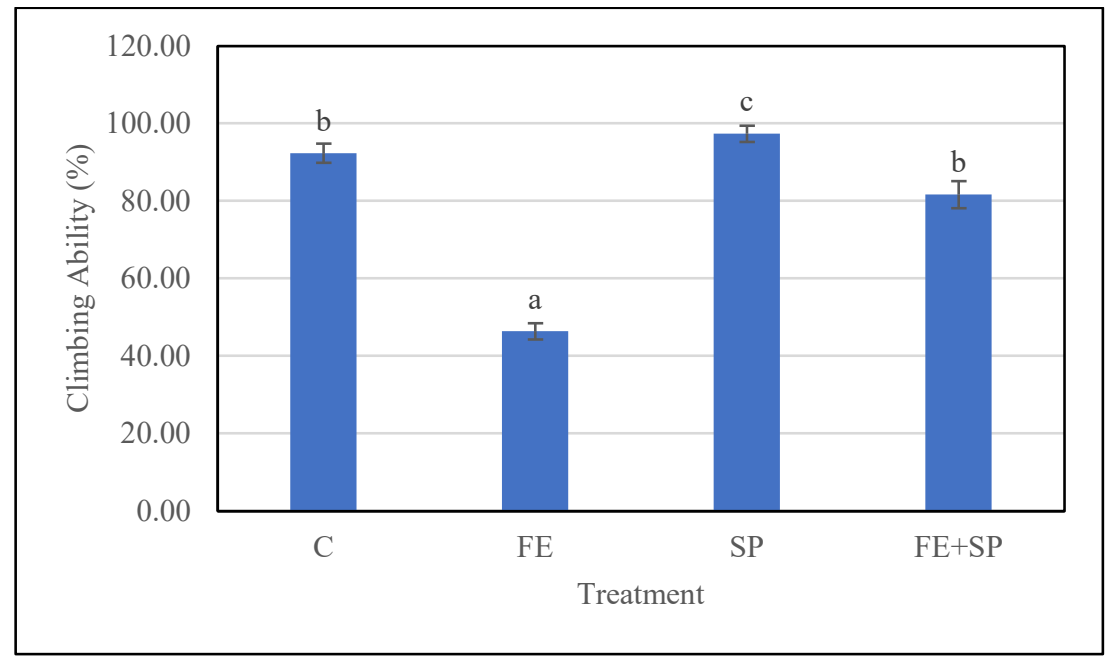

Fig. 3. Effect of SP $(120 \mu \mathrm{g} / \mathrm{mL})$ and FE $(3.5 \mathrm{mM})$ on negative geotaxis of fruit flies after 4 days of observation. Abbreviation of C: control, FE: FeSO4, SP: S. platensis extract. Data in the form of an average \pm Standard error. Values with different letters show significant differences at $\mathrm{p}<0.05$ (DMRT).

\subsection{Lipid Peroxidation}

FeSO4 administration showed an increase in malondialdehyde (MDA) levels in the fruit fly brain. S. platensis extracts (SP-treatment) can work in reducing MDA levels in fruit flies that have been exposed to FeSO4 (FE-treatment). MDA content is an indicator of the occurrence of oxidative stress indicated by the lipid peroxidation process on the head of the fruit fly (Figure.ure 4.) 


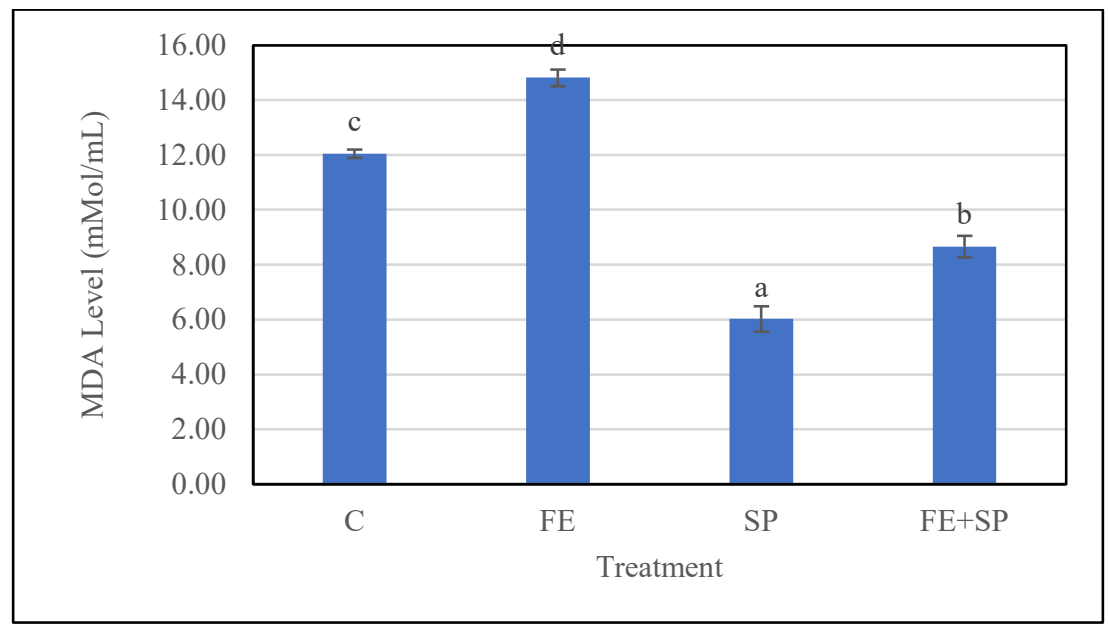

Fig. 4. Effect of SP $(120 \mu \mathrm{g} / \mathrm{mL})$ and FE $(3.5 \mathrm{mM})$ on MDA levels of fruit flies after 4 days of observation. Abbreviation of C: control, FE: FeSO4, SP: S. platensis extract and MDA : malondialdehide. Data in the form of an average \pm Standard error. Values with different letters show significant differences at $\mathrm{p}<0.05$ (DMRT).

\subsection{Dopamine Content}

From Figure.ure 5 it is shown that dopamine levels in the fruit fly brain are affected by all treatments. FeSO4 (FE-treatment) treatment was significantly $(\mathrm{P}<0.05)$ lowering dopamine levels while treatment of extract of $S$. platensis (SP-treatment) was able to maintain dopamine levels as in the control group (C). Furthermore, it is shown that $S$. platensis extract can increase dopamine levels in fruit flies that have been exposed to FeSO4 (FE+SP-treatment). 


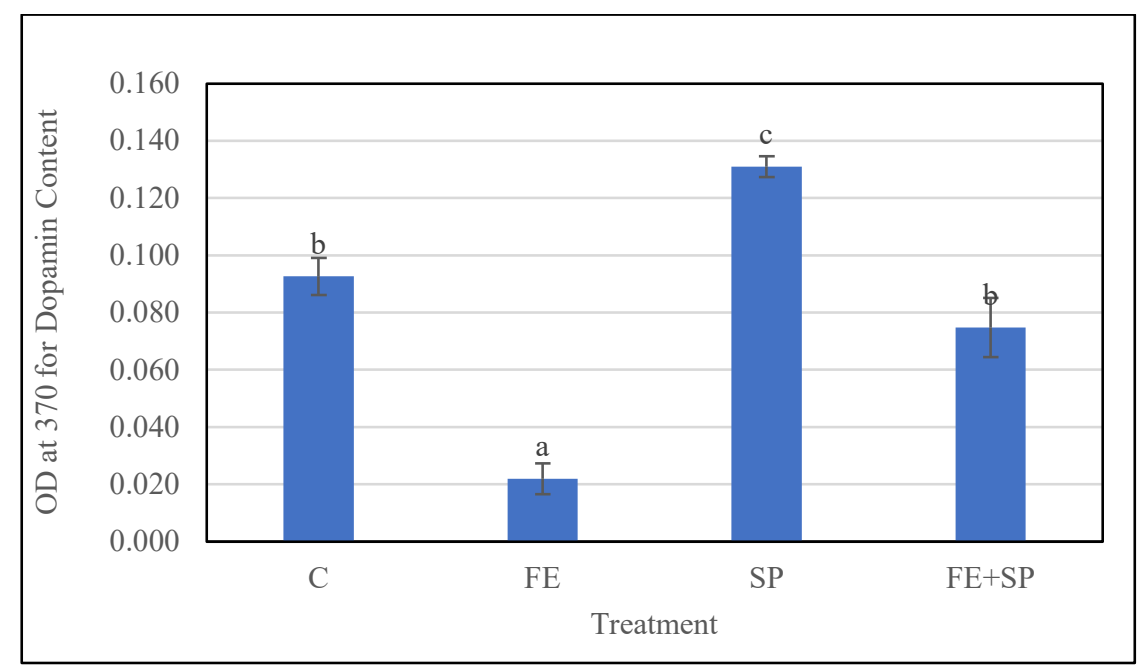

Fig. 5. Effect of SP $(120 \mu \mathrm{g} / \mathrm{mL})$ and FE $(3.5 \mathrm{mM})$ on dopamine levels of fruit flies after 4 days of observation. Abbreviation of C: control, FE: FeSO4, SP: S. platensis extract. Data in the form of an average \pm Standard error. Values with different letters show significant differences at $\mathrm{p}<0.05$ (DMRT)

\section{Discussion}

At present, fruit flies are used not only to obtain basic mechanical data but also to gain important knowledge of phytochemicals of natural materials that are able to Figure.ht neurotoxicity of neurodegenerative disorders [17]. $\mathrm{FeSO}_{4}$ has been well known as a highly toxic chemical substances which is often used as a model chemical because it is able to produce symptoms of Parkinson's disease that are important in fruit fly models [18]. Thus, both fruit flies as animal models and $\mathrm{FeSO}_{4}$ as neurotoxicity agents often obtain reproducible research and become a reference for many researchers throughout the world [19]. Incidentally, we often observe the ability of microalgae of various species to emerge FeSO4-induced oxidative stress, locomotor phenotype, and survival ability of fruit flies, these data are consistent with the findings of other researchers [20] and [16]. Interestingly, from this study, we demonstrated that the use of $S$. platensis extract can reduce the amount of mortality and locomotoric disorders induced by FeSO4. The great role of $S$. platensis microgreen extract in mediating rescue from fruit flies correlates with decreased oxidative stress (one of which decreases lipid peroxidation) (Figure.ure 4), increasing antioxidant defense (with increasing dopamine levels) (Figure.ure 5).

FeSO4 has shown its role as a cause of reduced ATP, oxidative damage and initial mortality [21]. According to [22] a decrease in dopamine levels occurs due to two factors: dopaminergic nerve loss or damage and increased dopamine compound oxidation. S. platensis extract has the potential as a neuroprotector agent which contains many antioxidant compounds such as polyphenols, chlorophyll and carotenoids to reduce free radicals produced by FeSO4. According to [23] the use of various antioxidant compounds can protect fruit flies from exposure to toxic compounds such as FeSO4 causing increased mortality, locomotor failure, and oxidative damage. In this study, we found that fruit flies treated with extracts of S. platensis were able to 
increase survivorship against $\mathrm{FeSO} 4$ which can be interpreted that extracts of S. platensis were very significant in survival pathways (Figure.ure 2). While FeSO4-only flies show locomotor defects, with giving $S$. platensis extract significantly improves locomotor performance (Figure.ure 3) by abrogate oxidative stress which does not damage dopaminergic neurons in the brain. Giving extract of S. platensis also significantly increases dopamine production in FeSO4induced fruit flies (Figure.ure 5). Furthermore, here extract of microgreens S. platensis shows its function as a neuromodulatory agent of dopaminergic neurons that produce dopamine compounds. Thus the symptoms of neurodegeneration caused by the presence of sources of free radicals such as $\mathrm{FeSO} 4$ can be delayed or reduced by the administration of extract of S. platensis on the diet of fruit flies.

\section{Conclusion}

We conclude that the extract of S. platensis included in the feed medium of fruit flies has been able to reduce or attenuate the power of FeSO4 toxicity. Likewise this extract of $S$. platensis demonstrates its real reliability as a neuroprotective agent against oxidative stress caused by $\mathrm{FeSO} 4$ as a neurotoxic agent. Based on the observations that have been made, it has been proven that there is a significant improvement of the locomotor phenotype and survival of fruit flies treated with extract of S. platensis. The use of extract of S. platensis can conclusively increase dopamine levels and reduce malondialdehyde levels in the head of fruit flies. Therefore S. platensis is an important therapeutic adjuvant in neurodegenerative disorders such as Parkinson's disease.

\section{Conflicts of Interest:}

Nill

\section{Acknowledgments:}

Authors acknowledges the support by Biology Department of Science and Technology of State Islamic University, Bandung, Indonesia

\section{References}

[1] P. D. Syad, A. N., Ajamohamed, B. S., Shunmugaiah, K. P., \& Kasi, "Neuroprotective effect. of the marine macroalga Gelidiella acerosa: identification of active compounds. through bioactivityguided fractionation. Pharm Biol, 54,” pp. 2073-2081, 2016.

[2] P. V. Singh, J. C., Kakalij, R. M., Kshirsagar, R.P., Kumar, B.H., Komakula, S.S., \& Diwan, "Cognitive effects of vanillic acid against streptozotocin-induced neurodegeneration in mice. Pharm Biol, 53,” pp. 630-636, 2015.

[3] C. Quintero-Espinosa, D., Jimenez-Del-Rio, M., \& Velez-Pardo, "Knockdown transgenic Lrrk Drosophila resists FeSO4-induced locomotor impairment and neurodegeneration: A therapeutic strategy for Parkinson's disease. Brain Research, 1657," pp. 253-261, 2016.

[4] M. N. Jhonsa, D. J., Badgujar, L. B., Sutariya, B. K., \& Saraf, "Neuroprotective effect of flavonoids against FeSO4 induced oxidative stress and neurotoxicity in Drosophila melanogaster. Cur Top Nutraceu Res, 14,” pp. 283-294, 2016.

[5] T. Niveditha, S., Ramesh, S. R. and Shivanandappa, "FeSO4-Induced Movement Disorder in Relation to Oxidative Stress-Mediated Neurodegeneration in the Brain of Drosophila 
Melanogaster Neurochem Res," 2017.

[6] A. K. Pal, P., and Ghosh, "Antioxidant, Anti-alzheimer and Anti-parkinson activity of Artemisia nilagirica Leaves with Flowering Tops UK Journal of Pharmaceutical and Biosciences.," pp. 12 23, 2018 .

[7] and Z. Y. Zhao, X., Zhang, M., Li, C., Jiang, X., Su, Y., "Review Article Benefits of Vitamins In the Treatment of Parkinson's Disease Hindawi Oxidative Medicine and Cellular Longevity.," pp. 1-14, 2019.

[8] M. C. Soares, J. J., Rodrigues, D.T., Gonçalves, M. B., Lemos, M.C., Gallarreta, M. S., Bianchini, "FeSO4 exposure-induced Parkinson's disease-like symptoms and oxidative stress in Drosophila melanogaster: Neuroprotective effect of Bougainvillea glabra Choisy. Biomed Pharma. 95," pp. 245-251, 2017.

[9] P. Vijayakumar S., Prabhu, S., Rajalakhsmi, S., Manoga, "Review on potential phytocompounds in drug develoSPent for Parkinson disease: A pharmaco informatic approach. Informatics in Medicine Unlocked," pp. 15-25, 2016.

[10] K. Yang, F., Wolk, A., Kansson, N.H., Pedersen, N.L., and Wirdefeldt, "Dietary Antioxidants and Risk of Parkinson's Disease in Two Population-Based Cohorts. Movement Disorders," 2017.

[11] F. F. de Fariasa, C.C., Maesa, M., Bonifácioa, K.L., Bortolascia, C.C., Nogueiraa, A.S., Brinholia, E. G. Matsumotoa, A.K., Nascimentoc, M.A., Melod, L.B., Nixdorfe, S.L., Lavadof, E.L., Moreiraa, and D. S. Barbosaa, "Highly specific changes in antioxidant levels and lipid peroxidation in Parkinson's disease and its progression: Disease and staging biomarkers and new drug targets. Neuroscience Letters 617," pp. 66-71, 2016.

[12] L. ska-Soczka, K.S., Zechini, L., and Zografos, "Validating the Predicted Effect of Astemizole and Ketoconazole Using a Drosophila Model of Parkinson's Disease ASSAY and Drug DeveloSPent Technologies," pp. 106-112, 2017.

[13] A. Askina, H., Yildiza, M. and Ayarb, "Special issue of the 3rd International Conference on Computational and Experimental Science and Engineering (ICCESEN 2016) Effects of Thymol and Carvacrol on Acetylcholinesterase from Drosophila melanogaster Acta Physica Polinica," 2017.

[14] V. Nikolova, G., Karamalakova, Y., Gadjeva, "Reducing oxidative toxicity of L-dopa in combination with two different antioxidants: an essential oil isolated from Rosa Damascena Mill., and vitamin C. Toxicology Reports 6.," pp. 267-271, 2019.

[15] and A. Yeung, A.W.K., Tzvetkov, N. T., El-Tawil, O.S., Bungău, S.G., Abdel-Daim, M.M. and A.G., "Antioxidants: Scientific Literature Landscape Analysis. Hindawi Oxidative Medicine and Cellular Longevity.," pp. 1-11, 2019.

[16] A. K. Kumar, A., Christian, P.K., Panchal, K., Guruprasad, B. R. \& Tiwari, "Supplementation of Spirulina (Arthrospira platensis) Improves Lifespan and Locomotor Activity in FeSO4-Sensitive DJ $1 \beta \Delta 93$ Flies, a Parkinson's Disease Model in Drosophila melanogaster,” Diet. Suppl., 2017.

[17] S. Siddique, Y.H. and Jyoti, "Alteration in biochemical parameters in the brain of transgenic Drosophila melanogaster model of Parkinson's disease exposed to apigenin Integrative Medicine Research," pp. 245-253, 2017.

[18] W. Ding. Y., Xin, C., Zhang, C., Lim, K., Zhang, H., Fu, Z., Li, L., and Huang, "Natural Molecules From Chinese Herbs Protecting Against Parkinson's Disease via Anti-oxidative Stress Frontiers in Aging. Neuroscience.," vol. 10, p. 246, 2018.

[19] I. Balakrishnan, R., Tamilselvam, K., Sulthana, A., Mohankumar, T., Manimaran, D. and N.E., "Attenuates Oxidative Stress and Behavioral Impairment in Rotenone-Induced Rat Model of Parkinson's Disease. International Journal of Nutrition, Pharmacology, Neurological Diseases," vol. XX, no. XX, 2018.

[20] M. A. Fadaka, A.O., Ajiboye, B.O., Adewale I., Ojo, O.A., Oyinloye, B.E., Okesola, "Significance of Antioxidants in the Treatment and Prevention of Neurodegenerative Diseases The Journal of Phytopharmacology.," pp. 75-83, 2019.

[21] A. and P. Silva, J., Alves C., Freitas, R., Martins, A., Pinteus, S., Ribeiro, J., Gaspar, H., Alfonso and R., "Antioxidant and Neuroprotective Potential of the Brown Seaweed Bifurcaria bifurcata in an in vitro Parkinson's Disease Model. Mararine Drugs," pp. 1-17, 2019. 
[22] M. . Muñoz, Y., Carrasco, C.M., Campos, J.D., Aguirre, P., and Marco T. Núñez, "Parkinson's Disease: The Mitochondria-Iron Link Hindawi Publishing Corporation Parkinson's Disease," pp. $1-21,2016$.

[23] J. L. Foti, S. C., Hargreaves, I., Carrington, S., Kiely, A. P., Houlden, H. \& Holton, "Cerebral mitochondrial electron transport chain dysfunction in multiple system atrophy and Parkinson's disease Scientific Reports.," 2019. 